degree of right ventricular hypertrophy (RVH) assessed and lung histology analysed for evidence of vascular remodelling. The lungs were stained with $\boldsymbol{\alpha}$-smooth muscle actin and the degree of distal muscularisation in vessels $<80 \mathrm{~mm}$ in diameter assessed. Results were analysed with appropriate statistical tests.

Results There was a significant difference in the RVSP between groups (control $37.09 \mathrm{~mm} \mathrm{Hg} \pm 5.09$ vs drug $20.59 \mathrm{~mm} \mathrm{Hg} \pm 3.19$; $\mathrm{p}=0.025$ ). There was less RVH (control 0.38 vs drug $0.25 ; \mathrm{p}=0.0032$ ) in the drug treated group (see Abstract S68 figure 1) and the total RV weights were also less (control $147 \mathrm{mg}$ vs drug $109 \mathrm{mg}$; $\mathrm{p}=0.018$ ). There was no difference in haematocrit between groups. There was less pulmonary vascular remodelling as indicated by a reduction of fully muscularised and an increase in non-muscularised vessels observed in the drug treated group $(p<0.001)$.

Conclusion We have shown in a chronic hypoxic model of $\mathrm{PH}$ that by inhibiting the p38 MAPK pathway in vivo the development of pulmonary hypertension can be prevented. This suggests that the p38 MAPK pathway could be a potential therapeutic target for $\mathrm{PH}$. Further studies are warranted, in particular to see if inhibition can reverse established disease.

\section{S69 SERUM OSTEOPROTEGERIN PREDICTS MORTALITY IN A PROSPECTIVE STUDY ON INCIDENT CASES OF PULMONARY ARTERIAL HYPERTENSION}

doi:10.1136/thoraxjnl-2011-201054b.69

${ }^{1} \mathrm{R}$ Condliffe, ${ }^{2} \mathrm{~J}$ Pickworth, ${ }^{2} \mathrm{~K}$ Hopkinson, ${ }^{2} \mathrm{~A}$ Hameed, ${ }^{3} \mathrm{~S}$ Walker, ${ }^{1} \mathrm{C}$ Elliot, ${ }^{2} \mathrm{~S}$ Francis, ${ }^{2} \mathrm{C}$ Newman, ${ }^{3} \mathrm{D}$ Crossman, ${ }^{3} \mathrm{~A}$ Morton, ${ }^{1} \mathrm{D}$ Kiely, ${ }^{2} \mathrm{~A}$ Lawrie. ${ }^{1}$ Royal Hallamshire Hospital, Sheffield, UK; ${ }^{2}$ University of Sheffield, Sheffield, UK; ${ }^{3}$ NIHR Sheffield Cardiovascular Biomedical Research Unit, Sheffield, UK

Background and Objectives Despite improvements in the overall management of Pulmonary Arterial Hypertension (PAH) the disorder still causes significant morbidity and mortality. Current treatments fail to reverse the disease, and clinical assessment does not always differentiate between, or reflect, the local pathogenesis within the heart or pulmonary circulation. Current proposed biomarkers, for example, brain natriuretic peptide (BNP and NT-proBNP), largely reflects myocardial rather than pulmonary vascular remodelling. Subsequently, there has been increasing interest in identifying a biomarker for PAH that can track with lung pathology, and treatment. Through our desire to understand disease pathogenesis, our studies in vitro and in animal models have identified osteoprotegerin (OPG) as a candidate biomarker. We have previously reported that OPG was elevated in a prevalent cohort of patients with IPAH. The aim of this study was to verify the utility of OPG as a biomarker for $\mathrm{PAH}$ in a second cohort of incident cases and assess the effect of treatment at follow-up visits.

Methods Serum samples were obtained from 35 patients with IPAH, 26 patients with CTD-PAH and 65 age-matched controls. Serum OPG concentrations were measured by ELISA, correlations with pulmonary haemodynamics, routine clinical biochemistry and prognostic significance were then assessed.

Results OPG concentrations were significantly elevated in IPAH (mean $4485 \mathrm{pg} / \mathrm{ml}$ ) and CTD-PAH $(3824 \mathrm{pg} / \mathrm{ml})$ compared to controls $(1749 \mathrm{pg} / \mathrm{ml})$. Concentrations of OPG correlated positively with pulmonary vascular resistance (PVR) and WHO functional class and negatively with the incremental shuttle walk test (ISWT). An OPG concentration above $4744 \mathrm{pg} / \mathrm{ml}$ predicted poorer survival. OPG was significantly lower in patients at follow-up after the commencement of targeted PAH therapies.

Conclusion PAH is characterised by elevated serum OPG and this correlates with functional class and PVR. Perhaps most importantly high serum levels of OPG predict a poor outcome. Further longitudinal work is required, and is currently underway to further validate these findings.

\section{S70 DIAGNOSTIC UTILITY AND PROGNOSTIC VALUE OF QUANTITATIVE CARDIAC MR INDICES IN PATIENTS WITH SUSPECTED PULMONARY HYPERTENSION}

doi:10.1136/thoraxjnl-2011-201054b.70

${ }^{1} \mathrm{~A} J$ Swift, ${ }^{1} \mathrm{~S}$ Rajaram, ${ }^{2} \mathrm{R}$ Condliffe, ${ }^{1} \mathrm{D}$ Capener, ${ }^{3} \mathrm{C}$ Hill, ${ }^{3} \mathrm{C}$ Davies, ${ }^{2} \mathrm{~J}$ Hurdman, ${ }^{2} \mathrm{C}$ Elliot, ${ }^{1} \mathrm{~J} \mathrm{M}$ Wild, ${ }^{2} \mathrm{D} \mathrm{G}$ Kiely. ${ }^{1}$ University of Sheffield, Sheffield, UK; ${ }^{2}$ Sheffield Pulmonary Vascular Disease Unit, Sheffield, UK; ${ }^{3}$ Radiology Department, Hull and East Yorkshire Hospitals NHS Trust, Sheffield, UK

Introduction and Objectives The aim of this study was to assess the clinical utility of quantitative MR indices of cardiac morphology and function in a large cohort of patients with pulmonary hypertension (PH).

Methods We retrospectively studied 233 consecutive patients with suspected $\mathrm{PH}$ who underwent cardiac MRI and right heart catheterisation (RHC) within $48 \mathrm{~h}$. Four chamber and short axis (SA) CINE images were acquired using cardiac gated multi-slice imaging with a steady state free precession sequence at $1.5 \mathrm{~T}$. The diagnostic and prognostic significance of quantitative measurements of right ventricular morphology and function were assessed.

Results Right ventricular end-diastolic mass index was the measurement with the strongest correlation with $\mathrm{mPAP}(\mathrm{r}=0.74)$ and the highest diagnostic accuracy for the detection of $\mathrm{PH}$ (area under the receiver operator curve of 0.91). During the mean follow-up of 18 months (0-36 months), 36 patients with $\mathrm{PH}$ died. Right ventricular ejection fraction $(\mathrm{p}=0.003)$, right ventricular stroke volume index $(p=0.03)$ and IVC size $(p=0.01)$ were the MR predictors of mortality across the subgroups of $\mathrm{PH}$. MR measurements of right ventricular ejection fraction $(p=0.004)$, right ventricular stroke volume index $(p=0.02)$, and left ventricular diastolic eccentricity index $(\mathrm{p}=0.005)$, all predicted mortality in patients with pulmonary arterial hypertension, Abstract S70 figure 1 below shows the Kaplan-Meier plots the MR predictors of mortality in PAH. IVC size $(p=0.018)$ was an independent predictor of mortality in the full cohort of patients with $\mathrm{PH}$, and diastolic eccentricity index $(p=0.037)$ was an independent predictor of adverse outcome in patients with PAH.
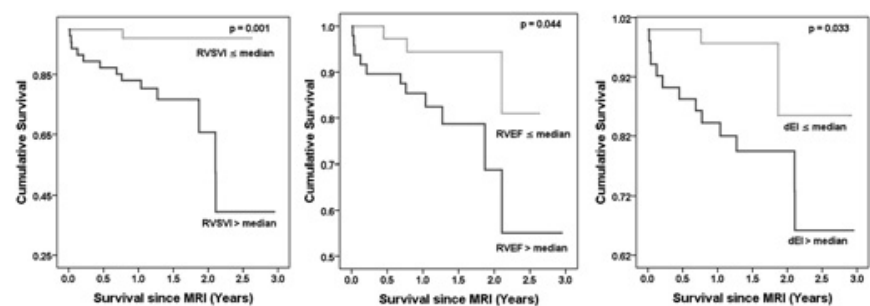

Abstract S70 Figure 1 Kaplan-Meier plots showing the survival curves for patients with PAH above and below the median value of MR derived right ventricular stroke volume index (RVSVI), right ventricular ejection fraction (RVEF) and diastolic eccentricity index (dEI).

Conclusion Cardiac MRI provides a comprehensive assessment of right ventricular morphology and function in patients with $\mathrm{PH}$. This study confirms the diagnostic and prognostic applicability of MRI in unselected patients with $\mathrm{PH}$ of varied aetiologies in a practical clinical setting.

S71 INFLUENCE OF AGE ON CLINICAL PHENOTYPES OF
INCIDENT IDIOPATHIC PULMONARY ARTERIAL
HYPERTENSION. RESULTS FROM THE PULMONARY
HYPERTENSION REGISTRY OF THE UK AND IRELAND

doi:10.1136/thoraxjnl-2011-201054b.71

${ }^{1} Y$ Ling, ${ }^{1} \mathrm{M}$ K Johnson, ${ }^{2} \mathrm{D}$ Kiely, ${ }^{2} \mathrm{R}$ Condliffe, ${ }^{2} \mathrm{C}$ Elliot, ${ }^{3} \mathrm{~S}$ Gibbs, ${ }^{3} \mathrm{~L}$ Howard, ${ }^{4} \mathrm{~J}$ PepkeZaba, ${ }^{4} \mathrm{~K}$ Sheares, ${ }^{5} \mathrm{P}$ Corris, ${ }^{5} \mathrm{~A}$ Fisher, ${ }^{5} \mathrm{~J}$ Lordan, ${ }^{6} \mathrm{~S}$ Gaine, ${ }^{7} \mathrm{G}$ Coghlan, ${ }^{8} \mathrm{~J}$ Wort, 